\title{
Formação de coordenadores pedagógicos em alimentação escolar: um relato de experiência
}

\author{
In-service training for pedagogical coordinators \\ in school nutrition: the report of an experience
}

\author{
Ligia Amparo da Silva Santos ${ }^{1}$ \\ Danilo Melo de Morais Carvalho ${ }^{1}$ \\ Amélia Borba Costa Reis ${ }^{1}$ \\ Lilian Barbosa Ramos ${ }^{1}$ \\ Maria do Carmo Soares de Freitas ${ }^{1}$
}

${ }^{1}$ Centro Colaborador em Alimentação e Nutrição do Escolar, Escola de Nutrição, Universidade Federal da Bahia. Rua Araújo Pinho 32, Canela. 40110-150

Salvador BA.

cecane@ufba.br

\begin{abstract}
This article describes the experience of in-service training for pedagogical coordinators of the Brazilian School Nutrition Program (PNAE) developed by the Cooperation Center for Student Food and Nutrition of the Federal University of Bahia (CECANE-UFBA). Participant observation and analysis of data documented in films, group discussion summaries, procedural and final evaluations and field diaries are used. The formative experiences were in 2011, in two municipalities in Bahia and Sergipe states, involving 118 pedagogical coordinators from 79 municipalities in both states. It was revealed that the objective of raising awareness and guiding pedagogical coordinators for mainstreaming the theme of food and nutrition has been achieved. The educational proposal was evaluated by the coordinators as " $\mathrm{dy}$ namic," "productive" and "interactive," promoting the exchange of experiences among participants. Despite considering the theme relevant, the pedagogical coordinators do not have sufficient training about food and nutrition to back their teaching practices. It is considered that their training has some limitations on the impact in the fieldwork, as the knowledge acquired night be dissipated when the coordinators return to their workplace due to the lack of ongoing training.

Key words School nutrition, Food and nutrition, Pedagogical coordinators, Food and nutrition education
\end{abstract}

Resumo Este artigo descreve a experiência de formação de coordenadores pedagógicos em Alimentação Escolar desenvolvida pelo Centro Colaborador em Alimentação e Nutrição do Escolar da Universidade Federal da Bahia. Utilizou-se da observação participante e análise de dados documentados em: filmagens, resumos de discussão grupal, avaliações processuais e finais e diários de campo. As experiências de formação foram em 2011, em dois municípios da Bahia e na capital de Sergipe, com 118 coordenadores pedagógicos de 79 municípios dos dois estados. Constatou-se que os objetivos da formação de sensibilizar e orientar os coordenadores para a transversalização do tema alimentação e nutrição no currículo escolar foram alcançados. A proposta educativa foi avaliada pelos participantes como "dinâmica", "produtiva" $e$ "interativa", promovendo trocas de experiências. Embora considerem um tema relevante, os coordenadores pedagógicos não têm formação suficiente nos conhecimentos de alimentação e nutrição para subsidiar as suas práticas pedagógicas. Considerase que a formação apresente limitações quanto a seu desdobramento no campo de trabalho, pois a sensibilização alcançada pode ser fragilizada na volta dos atores ao seu ambiente laboral por falta de uma formação permanente.

Palavras-chave Alimentação escolar, Alimentação e nutrição, Coordenadores pedagógicos, Educação alimentar e nutricional 


\section{Introdução}

Dada a importância do processo formativo dos educadores nos espaços escolares, Lima e Santos ${ }^{1}$ apontam que o coordenador pedagógico possui como importante atribuição favorecer o trabalho docente na escola, por meio da formação continuada de professores, devendo sensibilizá-los sobre seu saber-fazer pedagógico. Desta maneira, com a introdução recente desse profissional no cenário educacional brasileiro e alguma incongruência entre os objetivos e a prática de sua atuação, algumas iniciativas estão sendo tomadas para melhor delinear suas funções. Nesse sentido, em documento oficial da Prefeitura Municipal de Salvador ${ }^{2}$ foram listadas diretrizes para o exercício dessa coordenação em que se afirma que esta deve ser uma ação dinamizadora dos processos educativos, possibilitando a integração das dimensões política, pedagógica e administrativo-financeira da gestão escolar, a fim de otimizar o processo de ensino-aprendizagem para os alunos.

Neste sentido, entendendo a função articuladora do coordenador pedagógico na escola, o Centro Colaborador em Alimentação e Nutrição do Escolar da Universidade Federal da Bahia (CECANE-UFBA) aposta que sua formação junto a outros atores do Programa Nacional de Alimentação Escolar (PNAE) é relevante para potencializar a dimensão formativa dos educandos, como ainda orientando-os para as práticas alimentares saudáveis.

Nas normatizações do PNAE, proposições nessa direção podem ser percebidas a partir de 2006, com a Portaria Interministerial 1.010/2006, que define como primeiro eixo prioritário para a promoção da alimentação saudável nas escolas as ações de educação alimentar e nutricional; a Lei no 11.947/2009 e a Resolução FNDE no 38/ 2009 expõem em sua segunda diretriz que deve haver a inclusão da educação alimentar e nutricional no processo de ensino e aprendizagem, perpassando pelo currículo escolar, abordando o tema alimentação e nutrição e o desenvolvimento de práticas saudáveis, na perspectiva da segurança alimentar e nutricional ${ }^{3-5}$.

O Ministério da Educação e do Desporto (MEC) do Brasil, por seu turno, publicou em 1997 os Parâmetros Curriculares Nacionais $(\mathrm{PCN})^{6}$, na tentativa de estabelecer referências fundamentais para o projeto de formação do sujeito, inserindo o tema saúde, discutindo a importância da alimentação e nutrição neste processo. Nesta esteira, Santos ${ }^{7}$ destaca que o PNAE, coordenado pelo Fundo Nacional de Desenvol- vimento da Educação (FNDE), do Ministério da Educação, tem sofrido historicamente inúmeras alterações no seu aparato legal, propiciando cada vez mais a incorporação da promoção da alimentação saudável no ambiente escolar como uma importante meta.

Uma das iniciativas que merece destaque é o "Projeto Educando com a Horta Escolar", da FAO em parceria com o FNDE, que vem se constituindo como uma atividade educativa em alimentação e nutrição na escola, podendo ser visto como uma possibilidade em que docentes de todas as áreas e níveis de ensino desenvolvam atividades salutares e dinâmicas, envolvendo conhecimentos multidisciplinares, privilegiando o trabalho em grupo, a construção e a socialização de conhecimentos ${ }^{8}$.

Com a horta escolar propõe-se auxiliar em aulas práticas de disciplinas diversas, como língua portuguesa, ciências, geografia, história, envolvendo docentes e discentes, devendo fazer parte de projeto com objetivos e metodologias claros, proporcionando ainda que estes sejam avalia$\operatorname{dos}^{9}$ e implementados de modo que haja discussão integrada das temáticas da alimentação, nutrição, ambiente e currículo escolar, potencializando ações pedagógicas que visam: reflexões e mudanças nos hábitos alimentares dos escolares; adaptações de cardápios escolares às especificidades locais, inclusive no que se refere à introdução de alimentos da agricultura familiar no PNAE; ampliação das possibilidades de trabalhar com projetos de Educação Ambiental; melhoria da qualidade do trabalho pedagógico e no desempenho dos alunos no que se refere à aprendizagem e à lida com a alimentação.

Deste modo, o presente artigo objetiva relatar a experiência de formações de Coordenadores Pedagógicos no Programa de Formação Simultânea do PNAE, realizadas pelo CECANEUFBA no ano de 2011, indicando os seus limites e possibilidades.

\section{Método}

Para a descrição da experiência de formação realizada em 2011, utilizou-se da observação participante e análise de dados documentados ao longo dos encontros em: filmagens, resumos de discussão grupal, avaliações processuais e finais e diários de campo. Os dados produzidos foram sistematizados e analisados em articulação com o referencial teórico citado neste artigo. As experiências de formação ocorreram em dois muni- 
cípios do estado da Bahia e na capital de Sergipe envolvendo ao todo 118 coordenadores pedagógicos de 79 municípios dos dois estados. Os profissionais participantes foram todos advindos da rede pública de ensino.

\section{Resultado}

\section{Formação da Equipe do CECANE-UFBA}

A equipe composta pelos agentes de formação do CECANE-UFBA (agentes do PNAE) contou com a participação de nutricionistas, contadores, socióloga e pedagogo. A participação deste último foi destinada para otimizar a dinâmica da educação permanente da própria equipe e para colaborar na construção da proposta de formação dos coordenadores pedagógicos, realizando a interface entre os discursos do campo educacional com o da saúde.

A integração do grupo de mediadores da formação, o estabelecimento de diálogos, leituras, discussões e avaliações críticas de planos de trabalho, relatórios anteriores das ações do CECANE-UFBA, confrontados com referências bibliográficas possibilitaram compreender demandas, dificuldades e potenciais sobre a mesma, base para contextualizar a contribuição pedagógica na observação dos planos de formação dos atores do PNAE, colaborando para reflexão e aperfeiçoamento dos mesmos.

Dentro da proposta de formação dos agentes envolvidos no PNAE, realizaram-se encontros pedagógicos, nos quais eram estudados e discutidos criticamente temas como: educação permanente em saúde; aprendizagem significativa; conceito de educação e de educador; abordagens compreensivas para contextualizar a formação; formação enquanto experiência e indicadores de avaliação da formação, que melhor subsidiaram as atividades desenvolvidas com os coordenadores pedagógicos.

\section{O desenho metodológico da formação dos coordenadores pedagógicos}

A formação de Coordenadores Pedagógicos foi inspirada na aprendizagem significativa ${ }^{10}$, numa abordagem multirreferenciada ${ }^{11}$, crítica e dialógica ${ }^{12}$, pela necessidade de compreender com os aprendizes quais são os problemas, as demandas, os potenciais e as possibilidades apresentados sobre o contexto. A clareza de que a contextualização da proposta do PNAE só seria possível gradativamente a partir de associações com conhecimentos prévios dos participantes em relação às condições materiais e socioculturais é o que justifica a contribuição da aprendizagem significativa para a proposta de formação do PNAE.

A abordagem crítica depende ainda da relação segura e acolhedora entre educadores e educandos, para que seja possível aperfeiçoar a formação a partir das contribuições dos participantes, justificando avaliações processuais, a fim de otimizar a qualidade do processo de ensino e aprendizagem. Priorizou-se, ainda, usar abordagens metodológicas problematizadoras, estimulando a reflexão desses profissionais sobre suas realidades.

Assim, o objetivo delineado para a formação foi o de sensibilizar e orientar os coordenadores pedagógicos no uso de suas possibilidades de propor e construir, junto com a comunidade escolar, práticas educativas voltadas para a transversalização do tema alimentação e nutrição, enquanto projeto pedagógico.

No que tange aos conteúdos programáticos, estes tiveram por base os grandes eixos temáticos relacionados ao PNAE, a saber: o PNAE como estratégia para promoção da Segurança Alimentar e Nutricional; Direito Humano à Alimentação Adequada; Educação Alimentar e Nutricional no âmbito escolar e de projetos pedagógicos; Controle e Participação Social; Normas e Técnicas para gestão do PNAE; o PNAE e interfaces com outros programas e políticas públicas.

A complexidade dos conceitos de "saúde", "alimentação", "nutrição" e afins colocaram para os agentes da formação a necessidade de multirreferenciar as discussões, abordando tais noções a partir de dimensões culturais, políticas e geográficas influentes na construção social das práticas alimentares e desses conceitos. Tal discussão situou o tema da alimentação e nutrição no currículo escolar para além da promoção da alimentação saudável e da saúde, entendendo o ato de comer como um ato cultural e socializador, um marcador de identidade ${ }^{13}$.

O desenho metodológico proposto para a formação simultânea dos atores do PNAE consistiu em quatro etapas: a $1^{\text {a }}$ dedicou-se à atividade conjunta com todos os atores envolvidos na formação simultânea (nutricionistas, conselheiros e merendeiros da alimentação escolar mais coordenadores pedagógicos), compartilhando os objetivos da formação, seu histórico, objetivos e desafios do PNAE, relacionando-o aos temas Educação, Direito Humano à Alimentação Adequada (DDHA), Políticas Públicas e Segurança 
Alimentar e Nutricional (SAN). As $2^{\text {a }}$ e $3^{\text {a }}$ fases foram destinadas para atividades específicas com cada ator, com temas e conteúdos específicos; a $4^{\text {a }}$, por fim, trabalhou a atividade integrada promovendo um diálogo entre os atores, a fim de fortalecer a compreensão de todos na construção e qualificação do PNAE.

Foram empregadas avaliações processuais e finais em cada formação realizada, a fim de reorientar a prática de atividades. Ao final de cada dia da formação, uma reflexão foi estimulada a partir das questões: "O que aprendi hoje?", "O que poderei utilizar deste aprendizado no meu trabalho?". As falas foram registradas e discutidas no grupo. Pontuam-se aqui questões referentes aos aspectos metodológicos empregados, temas trabalhados, logística da formação e aplicabilidade de temas e conteúdos abordados, bem como a avaliação dos mediadores.

\section{O desenvolvimento das formações de coordenadores pedagógicos}

As formações de coordenadores pedagógicos ocorreram dentro do Programa de Formação de Atores do PNAE implementado pelo CECANEUFBA, com seu caráter simultâneo, em que participaram concomitantemente nutricionistas, conselheiros e merendeiros da alimentação escolar, além dos coordenadores pedagógicos.

$\mathrm{O}$ relato aqui posto corresponde às formações realizadas no ano de 2011, nos meses de julho, setembro e novembro, respectivamente, nos municípios de Barreiras e Juazeiro, ambos no estado da Bahia, e em Aracaju, capital de Sergipe, contando-se com a duração de 24 horas cada, realizadas em 03 dias consecutivos.

Cada uma dessas formações contou com a participação de coordenadores pedagógicos representantes dos municípios convidados, a saber, aqueles que compunham os territórios de identidade da cidade-sede de cada formação, e outros geograficamente mais próximos. Dessa forma, participaram coordenadores pedagógicos de 17, 24 e 38 distintos municípios nas formações de Barreiras, Juazeiro e Aracaju, respectivamente. Desta maneira, as experiências de formação envolveram ao todo 118 coordenadores pedagógicos de 79 municípios dos estados da Bahia e Sergipe.

Dentro desse caráter da simultaneidade, a formação de coordenadores pedagógicos teve como atividades específicas (referentes às $2^{\text {a }}$ e $3^{\text {a }}$ etapas citadas acima) o estímulo à reflexão desses profissionais sobre suas realidades e os as- pectos sociais, éticos, econômicos, políticos e culturais envolvidos na temática proposta, bem como o tema alimentação e nutrição como conteúdo escolar, a ser trabalhado de forma transversal, por entendê-los como essenciais para a saúde e para a formação integral do cidadão.

Desse modo, como estímulo à reflexão coletiva sobre o conhecimento prévio desses profissionais a respeito da relevância dos temas propostos, explorando suas nuances, limites e possibilidades, as formações específicas dos coordenadores pedagógicos iniciaram-se com o uso de questões como: "Qual a importância do tema saúde, alimentação e nutrição para a educação escolar?”; "Qual a importância do coordenador pedagógico para a educação do município?” e "Qual é o papel do coordenador pedagógico no PNAE?".

A partir da última questão acima, desenvolveu-se a discussão sobre a interface entre Ministérios e Programas, estabelecendo relações entre iniciativas do MEC e objetivos e diretrizes do PNAE e de políticas de saúde e de segurança alimentar e nutricional.

Seguindo-se a discussão e as reflexões acerca da alimentação e nutrição como temas transversais na Educação, focou-se no papel do coordenador pedagógico no PNAE a partir das suas próprias identificações no âmbito profissional, com a construção de plano de aula e planejamento pedagógico, partindo-se da justificativa para a questão "por que trabalhar o tema alimentação e nutrição no espaço escolar a partir da interdisciplinaridade e enquanto tema transversal e para que fazê-lo?".

Destaca-se que muito embora os coordenadores pedagógicos não necessariamente trabalhem diretamente com os estudantes esta proposta de construção de plano de aula visou estimular a sensibilidade desses atores para como o professor, na sua efetiva prática docente, poderia concretizar o tratamento transversal e transdisciplinar dos temas alimentação e nutrição.

Por fim, como proposta para se iniciar uma atividade envolvendo outro ator que contribuísse para a discussão dessa temática, fez-se, na última formação realizada em 2011, uma atividade de integração entre Coordenadores Pedagógicos e Nutricionistas. Mediada por agentes do PNAE do CECANE-UFBA, grupos desses profissionais desenvolveram propostas de ações educativas com os temas alimentação e nutrição, tendo por base casos com contextos socioeconômico, culturais e de saúde.

Os recursos utilizados para a formação foram textos e crônicas jornalísticas sobre o tema, 
projeções fílmicas, além de apresentações produzidas com referências bibliográficas e normativas do PNAE e temas afins.

\section{Analisando a Experiência de Formação}

$\mathrm{Na}$ apresentação da proposta do curso aos coordenadores pedagógicos sua especificidade ganhou mais evidência, mas também emergiu a estranheza dos presentes quanto ao que fariam os coordenadores pedagógicos, profissionais da educação, em um evento que trataria de alimentação escolar. Houve, inclusive, a interpretação equivocada quanto à representação dos municípios nos eventos, fazendo-se presentes outros que não coordenadores pedagógicos e sim gestores da alimentação escolar e gestores municipais de educação.

Esses fatos levam a refletir sobre o distanciamento das ciências da saúde, alimentação e nutrição (onde se prioriza o estudo da alimentação a partir do modelo biomédico) e das ciências humanas (onde prioritariamente se situa a área da Educação), supondo-se ainda que os temas alimentação e nutrição estejam distantes de serem tratados transversalmente nos Projetos Pedagógicos.

Por outro lado, alguns coordenadores demonstraram uma compreensão de que o tema era relevante e que deveria ser tratado na escola. Em parte, pode atribuir-se a isso ações, campanhas e programas especialmente do Ministério da Saúde divulgando dados epidemiológicos da população brasileira no que tange ao estado nutricional, particularmente de crianças e adolescentes, associando-os às práticas alimentares destes grupos etários.

$\mathrm{Na}$ etapa da formação em que questões foram postas para substanciar o debate sobre a relevância do tema saúde na formação dos educandos e o papel do coordenador na proposta do PNAE, os educadores apontaram a educação como fundamental para a formação de cidadãos críticos, reflexivos, conscientes e humanizados, facilitando a construção de conhecimentos e sendo o veículo de transformação da realidade social, histórica e cultural.

A educação em saúde, por sua vez, foi entendida como essencial para a formação plena do homem, promovendo sua possibilidade de autocuidado, tendo uma perspectiva crítica sobre o tema, para reflexões e intervenções individuais e/ ou coletiva sobre fatores condicionantes e determinantes do processo saúde/doença e da promoção da saúde.

Nos discursos dos coordenadores pedagógicos, em sua maioria, a saúde apareceu definida se- melhante a como o faz a Organização Mundial de Saúde (OMS), como um estado de bem estar mental, social e físico, e não apenas ausência de doença, sendo apontada, ainda, como pré-requisito para qualidade de vida e prevenção de doenças ${ }^{14}$.

Destaca-se que este próprio conceito da OMS tem sofrido críticas, por apontar para uma inatingível perfeição, baseando-se, ainda, em avaliações externas, objetivas e teoricamente mensuráveis ${ }^{15}$. Observa-se, entretanto, que essa própria definição de saúde trazida pelos educadores pareceu fragilizada no decorrer das demais práticas desenvolvidas na formação, apresentando sua compreensão mais próxima à ausência de doenças, em uma concepção predominantemente biológica.

Percebeu-se que mesmo a compreensão de "promoção de saúde" nos discursos dos coordenadores pedagógicos associou-se a transformações comportamentais individuais e ligadas à adoção de distintos estilos de vida, sofrendo influência apenas de aspectos culturais ou etários, semelhante àquela apontada por Buss apud Santos $^{16}$ sobre seu trajeto histórico e conceitual da promoção da saúde, cabendo, entretanto, estabelecer-se políticas públicas intersetoriais que promovam práticas saudáveis aos indivíduos e para estes nos espaços coletivos, quer públicos quer privados.

Apesar de parecerem sensibilizados quanto à importância de trabalhar a alimentação e a nutrição para a promoção da alimentação saudável e da saúde, os coordenadores pedagógicos apontaram que seu conhecimento a respeito destes era insuficiente, carecendo de educação continuada e de construções teórico-metodológicas que subsidiem as ações de educação alimentar e nutricional.

$\mathrm{Na}$ etapa da formação dedicada a detalhar as demandas educativas do PNAE, suas bases legais, histórico e as intenções da formação para coordenadores pedagógicos, emergiram discussões iniciais muito mais associadas à gestão de recursos deste programa. Foram apontadas veementemente a dificuldade prática em constituirse diálogos entre os distintos atores do PNAE, exemplificada pelo desconhecimento por parte da comunidade escolar a respeito de elaboração do cardápio da alimentação escolar, as precárias estruturas físicas para a elaboração e a distribuição das refeições e a falta de iniciativa articulada e dialogada para melhoria dessas condições.

A atividade dedicada à reflexão sobre práticas alimentares e saúde promoveu discussões e reflexões sobre concepções de alimento e alimentação saudáveis, base para discutir a construção 
do saudável enquanto aproximação de perspectivas e diálogos, considerando as múltiplas influências que possibilitam a construção daquelas noções. Quanto a essa formulação sobre o saudável, Azevedo ${ }^{17}$ aponta que o próprio conceito de dieta saudável é passível de mudanças a depender do contexto histórico-cultural, não cabendo em um consenso científico e biológico, pois como afirmam antropólogos e sociólogos citados por Santos ${ }^{18}$, a relação entre os seres humanos e a alimentação é complexa, extrapolando o domínio puramente biológico, devido ao fato de que práticas alimentares são construídas socialmente e tornam-se marcas identitárias.

Silva $^{19}$, ao referir-se sobre o conceito do saudável por profissionais da saúde, chama a atenção que estes devem "auferir conhecimentos e habilidades que potencializem seu papel de agente de saúde a partir do entendimento de que o processo é biocultural", o que deve ser enfatizado não apenas aos profissionais da saúde em si, mas aos da educação, que também educam para a saúde.

Entretanto, na atividade prática desenvolvida por coordenadores pedagógicos percebeu-se que a sua primeira perspectiva sobre o saudável restringia-se a uma dimensão dietoterápica e prescritiva, circunscrita ao que os escolares deveriam comer e não comer, considerando as composições nutricionais dos alimentos, as quais determinavam o que seria saudável e não saudável, de uma maneira dicotômica e excludente: ou é saudável ou não é saudável.

$\mathrm{Na}$ proposta de desenvolvimento de planos de aulas e planos de coordenação pedagógica articulando-se transversalmente a alimentação e nutrição, percebeu-se que nos primeiros foi recorrente aquela opção que inseria classificação e valorização de alimentos na sua qualidade de "saudável" e "não saudável" na perspectiva de sua composição nutricional, ratificando a percepção apontada anteriormente, de que as concepções biomédicas ainda prevalecem quando se pensa sobre a temática alimentação e nutrição.

Já na construção dos planos pedagógicos foi frequente a ideia de partilhar a intenção de introduzir o tema alimentação e nutrição nos projetos pedagógicos com gestores municipais da educação, outros coordenadores pedagógicos dos municípios ali representados, além da comunidade escolar (docentes, merendeiros e pais de alunos), referenciando os PCN e as normas do PNAE. Além dessa, o envolvimento dos alunos também foi contemplado, mais especificamente propondo-se que estes pesquisassem a respeito de hábitos e práticas alimentares para, então, tra- balhar-se com a discussão e as propostas de mudanças do cardápio da alimentação escolar.

Nestas produções, entretanto, identificou-se o caráter pontual das atividades propostas, e em poucas foi encontrado o interesse sobre práticas alimentares dos alunos, apesar da discussão sustentada nas formações sobre a importância de contextualizar as propostas pedagógicas.

A atividade integrada entre coordenadores pedagógicos e nutricionistas retratou a dificuldade existente para estabelecimento de diálogo entre esses atores, sendo demarcado em alguns grupos que a atividade proposta de planejamento das ações educativas com a temática da alimentação e nutrição ficou mais sob a responsabilidade do nutricionista e dentro de uma perspectiva biomédica. A avaliação metodológica desta atividade pelos participantes foi de que, ao invés do CECANE-UFBA apresentar casos contextualizados, deveria aproveitar situações de suas próprias realidades como base para essa discussão. Apesar do curto tempo destinado a esta atividade, foi finalizado o esboço de planos das ações educativas, tendo os participantes como possíveis multiplicadores da proposta do PNAE dentro de suas realidades.

Em suma, estas propostas pedagógicas construídas pelos participantes das formações ministradas pelo CECANE-UFBA se configuram como documentos de referência para compreender como os coordenadores pedagógicos se apropriam dos conteúdos, se foram sensibilizados e como reagem à proposta do curso, ao apresentarem seus planejamentos. Nas três formações, ficou evidente que propor a construção grupal de planos de aula e esboço de projetos pedagógicos que incorporam a temática da alimentação e nutrição, de forma transversal e interdisciplinar, possibilita rica interação entre os participantes do curso, com trocas de experiências e tempestade de ideias que organizam sugestões possíveis de viabilizarem a educação alimentar e nutricional nas escolas.

\section{Conclusão}

Ao levar em conta que o objetivo da formação de coordenadores pedagógicos foi o de sensibilizar e orientar os mesmos no uso de suas possibilidades de propor e construir, junto com a comunidade escolar, práticas educativas voltadas para a transversalização do tema da alimentação e nutrição, enquanto projeto pedagógico, pode-se considerar que o mesmo foi alcançado em grande parte. A proposta educativa da formação foi con- 
siderada por esses atores como "dinâmica", "produtiva" e "interativa", promovendo trocas de experiências entre os participantes, o que reforça a importância de se conservar e aperfeiçoar o modo como vem sendo desenvolvida a formação.

O fato dos participantes declararem-se sensibilizados indica possível motivação para a incorporação da proposta na prática pedagógica. Porém, é importante ressaltar que cursos de formação desta natureza apresentam limitações quanto a seu desdobramento, pois a sensibilização alcançada pode ser fragilizada no retorno dos atores ao seu cotidiano de trabalho, por falta de uma formação permanente. Isto indica a necessidade de investigação para compreender até que ponto a formação contribui para aperfeiçoar o trabalho dos coordenadores pedagógicos.

Sobre a última formação de 2011, salienta-se que a experiência de integrar coordenadores pedagógicos e nutricionistas para o planejamento de ações educativas foi avaliada como positiva, pois possibilitou a integração de diferentes atores e a possível continuidade dos planos esboçados nos espaços de trabalho.
Observou-se ainda que, apesar das discussões realizadas com a proposta de ampliar a compreensão sobre o fenômeno da alimentação e nutrição, nas atividades práticas de elaboração dos planos de aula, a perspectiva biomédica prevalecia. Deste modo, salienta-se que diretrizes teóricas e práticas que coadunem os objetivos educacionais da escola e os objetivos da promoção da alimentação saudável demandam considerar a escola como um espaço de diversidade, no qual diferentes práticas alimentares convivem simultaneamente. $\mathrm{O}$ entendimento de que as práticas alimentares saudáveis são produtos da cultura aponta para uma reflexão sobre a relação do homem com o comer ao longo da história da humanidade. Estes múltiplos discursos sobre o comer, a alimentação e a nutrição precisam ser dialogados e as diversidades valorizadas.

Destaca-se a relevância de estimular os processos de formação dos coordenadores pedagógicos em torno da temática proposta, articulando com políticas públicas interssetoriais que forneçam subsídios teórico-metodológicos a fim de colaborar para a inserção do tema alimentação e nutrição nos projetos pedagógicos.

\section{Colaboradores}

LAS Santos, DMM Morais Carvalho e ABC Reis trabalharam na concepcão do projeto, delineamento metodológico e na elaboração do artigo. LB Ramos e MCS Freitas participaram da concepção do ensaio e da revisão crítica do artigo. 


\section{Referências}

1. Lima PG, Santos SM. O coordenador pedagógico na educação básica: desafios e perspectivas. Educere et Educare - Revista de Educação 2007; 4(2):1809-5208.

2. Prefeitura Municipal de Salvador. Coordenador Pedagógico: traçando caminhos para sua prática educativa. Salvador: SMEC/CENAP; 2008. [acessado 2012 fev 24]. Disponível em: http://www.smec.salvador. ba.gov.br/site/documentos/espaco-virtual/espacocenap/publicacoes/coordenador $\% 20$ pedagogico \%20-\%20tra\%C3\%A7ando\%20caminhos\%20para\% 20sua\%20pratica.pdf

3. Brasil. Portaria Interministerial no 1.010 de 08 de maio de 2006. Institui as diretrizes para a promoção da alimentação saudável nas escolas de educação infantil, fundamental e nível médio das redes públicas e privadas, em âmbito nacional. Diário Oficial da União 2006; 9 maio.

4. Brasil. Lei $n^{\circ} 11.947$ de 16 de junho de 2009. Dispõe sobre o atendimento da alimentação escolar e do Programa Dinheiro Direto na Escola aos alunos da educação básica; altera as Leis nos 10.880, de 09 de junho de 2004, 11.273, de 6 de fevereiro de 2006 11.507, de 20 de julho de 2007; revoga dispositivos da Medida Provisória no 2.178-36, de 24 de agosto de 2001, e a Lei no 8.913, de 12 de julho de 1994; e dá outras providências. Diário Oficial da União 2009; 17 jun.

5. Brasil. Resolução no 38 de 16 de julho de 2009 . Dispõe sobre o atendimento da alimentação escolar aos alunos da educação básica no Programa Nacional de Alimentação Escolar - PNAE. Diário Oficial da União 2009; 17 jul.

6. Ministério da Educação e do Desporto. Parâmetros curriculares nacionais: língua portuguesa ( $1^{\mathrm{a}}$ à $4^{\mathrm{a}}$ série). Brasília: MEC; 1997.

7. Santos LAS. O fazer educação alimentar e nutricional: algumas contribuições para reflexão. Cien Saude Colet 2012; 17(Supl. 2):453-462.

8. Morgado FS, Santos MAA. A horta escolar na educação ambiental e alimentar: experiência no projeto Horta Viva nas escolas municipais em Florianó polis. Revista Eletrônica de Extensão da Universidade Federal de Santa Catarina 2008; (6):1-10. [periódico na Internet]. 2008. [acessado 2012 fev 24]. Disponível em: http://www.rebrae.com.br/experiencias/ A_horta_escolar.pdf
9. Trigo M, Silva MEMP. Educação nutricional na comunidade: Projeto de Hortas como veículo de Educação Nutricional. In: Garcia RWD, CervatoMacuso AM, organizadores. Mudanças alimentares e educação nutricional. Rio de Janeiro: Guanabara Koogan; 2011. p. 276-285.

10. Moreira MA. Aprendizagem significativa. Brasília: Editora Universidade de Brasília; 1999.

11. Macedo RS. Compreender-mediar a formação: o fundante da educação. Brasília: Liber Livro; 2010.

12. Freire P. Pedagogia do oprimido. 17a Edição. Rio de Janeiro: Paz e Terra; 1987.

13. Fischler C. L'homnivore. Paris: Odile Jacob; 1991.

14. Sclyar M. História do conceito de saúde. Physis: Revista de Saúde Coletiva 2007; 17(1):29-41.

15. Santos SMC. Alimentação e nutrição: um diálogo com os princípios de qualidade de vida. In: Freitas MCS, Fontes GAV, De Oliveira N, organizadores. Escritas e narrativas sobre alimentação e cultura. Salvador: EDUFBA; 2008. p. 261-275.

16. Santos LAS. Educação alimentar e nutricional no contexto da promoção de práticas alimentares saudáveis. Revista de Nutrição 2005; 18(5):681-692.

17. Azevedo E. Reflexões sobre o risco e o papel da ciência na construção do conceito de alimentação saudável. Revista de Nutrição 2008; 21(6):717-723.

18. Santos LAS. O corpo, o comer e a comida: um estudo sobre as práticas corporais alimentares cotidianas a partir da cidade de Salvador-Bahia. Salvador: EDUFBA; 2008.

19. Silva DO. Reflexões conceituais e de profissionais de saúde sobre a promoção da alimentação saudável. In: Garcia RWD, Cervato-Macuso AM, organizadores. Mudanças alimentares e educação nutricional. Rio de Janeiro: Guanabara Koogan; 2011. p. 164-172.

Artigo apresentado em 03/01/2013

Aprovado em 05/01/2013

Versão final apresentada em 08/01/2013 